\title{
Surfactant effects on the microstructure and electrochemical properties of emulsion-derived lithium cobalt oxide powders
}

\author{
Chung-Hsin Lu*, Po-Ying Yeh \\ Department of Chemical Engineering, National Taiwan University, Taipei, Taiwan, People's Republic of China
}

Accepted 8 March 2001

\begin{abstract}
The surfactant effects on the microstructure and electrochemical properties of lithium cobalt oxide $\left(\mathrm{LiCoO}_{2}\right)$ powders prepared from emulsion-derived precursors have been investigated in this study. The single-phase $\mathrm{LiCoO}_{2}$ powders with $\mathrm{R} \overline{3} \mathrm{~m}$ structure are successfully prepared. The crystallinity and particle size of the obtained powders are varied with the amount of the surfactant used for preparing the emulsion solution. Reducing the amount of the surfactant results in improving the crystallinity and slightly decreasing the particle size of $\mathrm{LiCoO}_{2}$ powders. The $\mathrm{LiCoO}_{2}$ powders prepared from the emulsion with a small amount of the surfactant added exhibit high discharge capacity and high coulomb efficiency, as well as good cyclability. The crystallinity of $\mathrm{LiCoO}_{2}$ powders is considered to play an important role in determining the electrochemical properties. (C) 2001 Elsevier Science B.V. All rights reserved.
\end{abstract}

Keywords: Electrochemical properties; Oxide powders; Emulsions

\section{Introduction}

Lithium ion batteries have been attracting considerable attention in the past decade in the field of rechargeable batteries because they exhibit high operation voltage, high specific density, steady discharging properties, little memory effect, and long cycle life [1-3]. For achieving the best performance of lithium ion batteries, selecting the right cathode materials plays an important role. Lithium cobalt oxide $\left(\mathrm{LiCoO}_{2}\right)$ has the advantages of superior cyclability, high energy density, and thermal stability therefore, it has become the most commonly adopted cathode material and is extensively used in a wide range of portable electronic appliances [4-6].

Many efforts have been devoted to investigate the crystal structure and electrochemical properties of $\mathrm{LiCoO}_{2}$ [7-9]. In most studies, $\mathrm{LiCoO}_{2}$ powders are prepared by the solid-state reaction. In this type of process, intensive mixing of reactants is necessary, and calcination at elevated temperatures for a prolonged period of time $(10-20 \mathrm{~h})$ is required $[10,11]$. Due to the

\footnotetext{
* Corresponding author. Fax: + 886-2-23623040.
}

E-mail address: chlu@ccms.ntu.edu.tw (C.-H. Lu). prolonged calcination, the formed powders experience serious grain-coarsening, and the stoichiometry of the obtained powders is difficult to control due to the evaporation of lithium species. In order to solve the above problems occurring in the solid-state reaction, various kinds of soft-chemistry processes have been investigated recently for synthesizing $\mathrm{LiCoO}_{2}$ powders. The sol-gel process [12,13] and the evaporation method [14], for example, have been studied. These processes can improve the reactivity of the precursors and control the morphology of $\mathrm{LiCoO}_{2}$ powders. In our earlier study, a new solution process - a water-in-oil emulsion process has been developed for synthesizing $\mathrm{LiCoO}_{2}$ powders [15]. This emulsion process not only significantly shortens the reaction time for preparing $\mathrm{LiCoO}_{2}$, but also decreases the particle size of $\mathrm{LiCoO}_{2}$ to submicron order. In the present study, the effects of the surfactant used for preparing the emulsion solution on the microstructure and crystallinity of $\mathrm{LiCoO}_{2}$ powders are further investigated. The electrochemical behavior of the obtained powders used as cathodes in the test cells of lithium-ion batteries are examined. The relation between the crystallinity and the electrochemical properties is discussed. 


\section{Experimental}

In the emulsion process, equal molar amounts of lithium and cobalt acetates were mixed in the de-ionized water to prepare the aqueous phase. The concentration of total cations in the aqueous phase was set to be $1.0 \mathrm{M}$. After the reactants were completely dissolved, kerosene (the oil phase) was added into the aqueous solution, followed by adding a surfactant-sorbitan monooleate to stabilize the water-in-oil emulsion solution. The water/oil volume ratio was fixed as 1:5. The volume percent of the surfactant in the mixed water-oil solution was varied from 5 to $15 \%$. The prepared emulsion solution after thoroughly mixing was dropped into hot kerosene for evaporating the aqueous phase, and then a gel was produced. After drying the gel at $300^{\circ} \mathrm{C}$ for $1 \mathrm{~h}$, the precursors of $\mathrm{LiCoO}_{2}$ were obtained. The precursors were calcined at $800^{\circ} \mathrm{C}$ for $1 \mathrm{~h}$ for synthesizing $\mathrm{LiCoO}_{2}$. The calcined specimens were examined by X-ray powder diffraction to identify the formed phases and crystallinity. Microstructures and particle sizes of the obtained powders were observed by scanning electron microscopy (SEM). Electrochemical behavior of the obtained $\mathrm{LiCoO}_{2}$ powders was examined in a two-electrode cell. The electrochemical cell consisted of a $\mathrm{LiCoO}_{2}$-based composite as the positive electrode, $\mathrm{Li}$ foil as the negative electrode, and an electrolyte of $\mathrm{LiPF}_{6}$ in EC/DMC system. The cell was cycled at 1/5 C-rate within the potential range of 3-4.2 V.

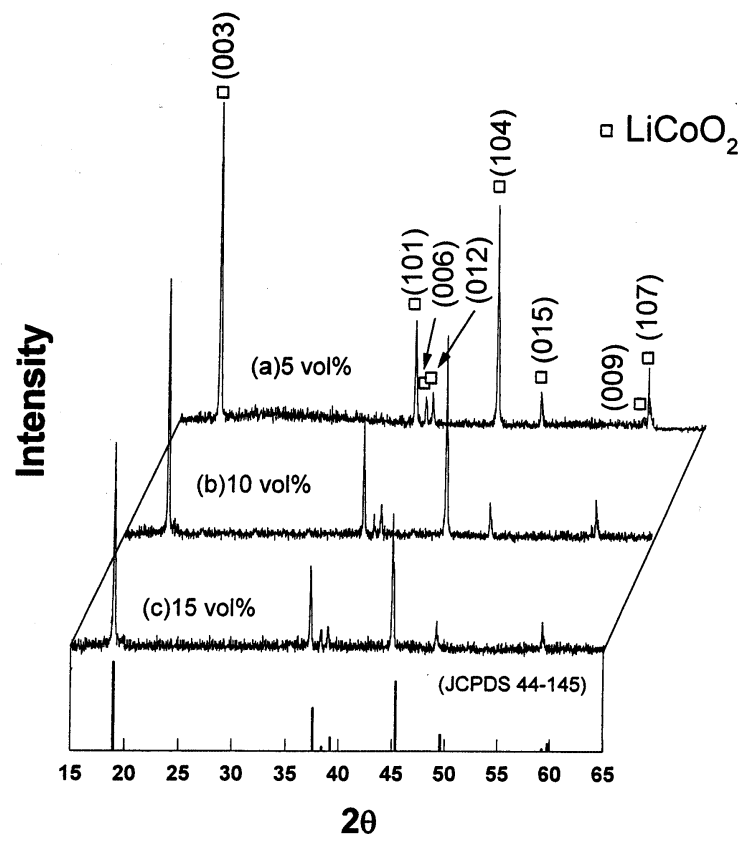

Fig. 1. X-ray diffraction patterns of the $800^{\circ} \mathrm{C}$-calcined $\mathrm{LiCoO}_{2}$ powders derived from the emulsion process using various amounts of the surfactant.
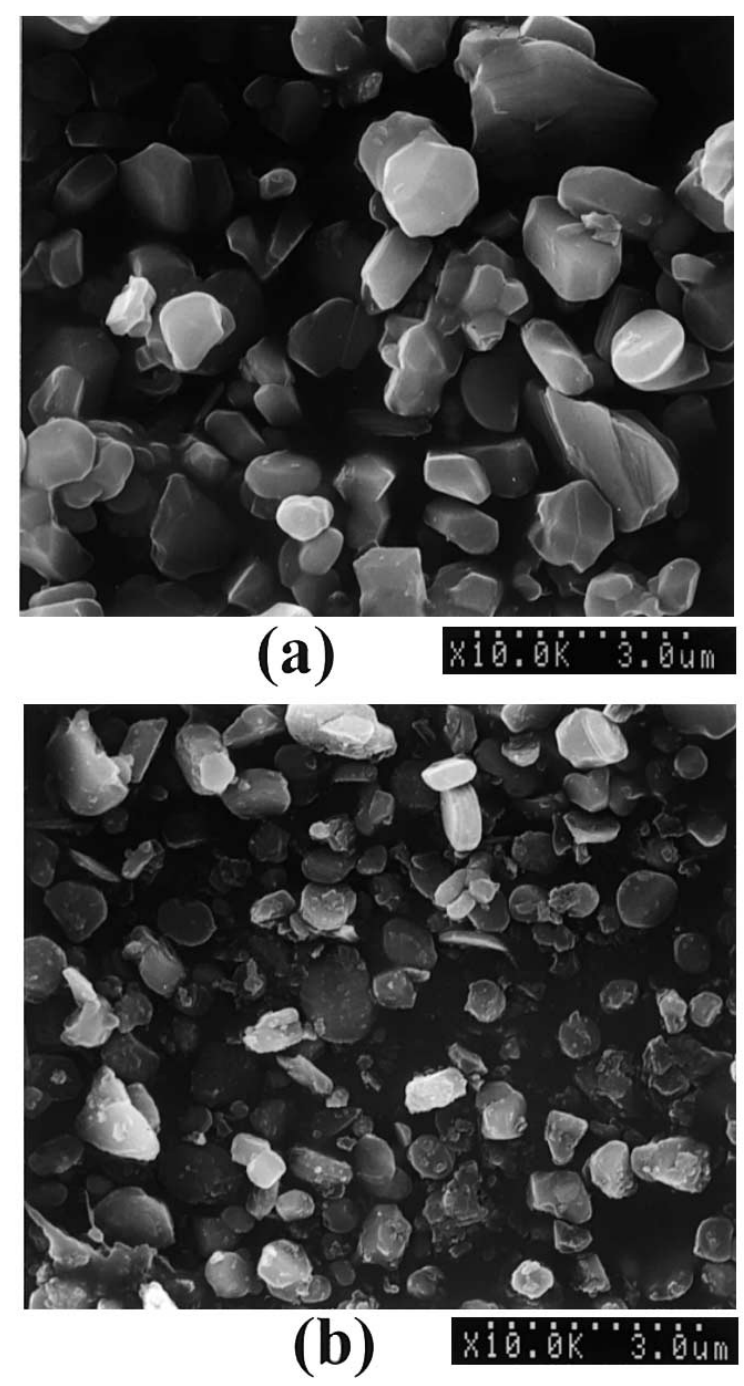

Fig. 2. Scanning electron micrographs of $\mathrm{LiCoO}_{2}$ powders derived from the emulsion process using various amounts of the surfactant ((a) 5 vol. $\%$ and (b) 15 vol. $\%$ ).

\section{Results and discussion}

The XRD diffraction patterns of the $800^{\circ} \mathrm{C}$-calcined precursors prepared from the emulsion solutions with various amounts of the surfactant added are shown in Fig. 1. All three XRD patterns show great consistency with that reported in JCPDS file [16]. The diffraction peaks in the XRD patterns were indexed to a hexagonal symmetry, which confirms that the obtained $\mathrm{LiCoO}_{2}$ powders exhibit the $\mathrm{R} \overline{3} \mathrm{~m}$ structure (high-temperature polymorph). Although the peak positions in the above three patterns are identical, the intensities of diffraction peaks are different. It is found that the intensity of diffraction peak increases with a decrease in the amount of the surfactant-sorbitan monooleate used in w/o emulsion. It indicates that the crystallinity of $\mathrm{LiCoO}_{2}$ powders is enhanced by decreasing the amount of the surfactant. 
Fig. 2 shows the effects of the surfactant amount on the morphology of the obtained $\mathrm{LiCoO}_{2}$ powders. All obtained powders exhibit a well-dispersed state. When 5 vol.\% surfactant was used, the prepared sample exhibited well-developed facet planes (as shown in Fig. 2a). When the amount of the surfactant increased, the morphology of $\mathrm{LiCoO}_{2}$ powders became rounded (see Fig. 2b). The variation in the morphology seemingly correlates with the variation in the crystallinity of the powders. The particle size of the synthesized powders was further analyzed by a particle size analyzer. The average particle sizes are $1.47,1.42$, and $1.26 \mu \mathrm{m}$ for the powders prepared with the added amount of the surfactant equal to 5,10 , and $15 \%$, respectively. It reveals that the particle size slightly decreases as the amount of the surfactant increases.

Generally, the correlation between the size of the particle obtained from the emulsion solution and that of the emulsion droplet can be expressed as below [17]:

$D=d\left(\frac{\rho(1-P)}{M C}\right)^{1 / 3}$

where $D$ and $d$ are the diameters of the emulsion droplet and the particle, $\rho$ the theoretical density of the solid particle, $P$ the porosity of the particle, $C$ the cation concentration of the aqueous phase, $M$ the molecular weight of the particle. Since the values of $\rho$, $C$ and $M$ are the same for each specimen in this study, the diameter (size) of the particle will be proportional to the diameter (size) of the emulsion droplet assuming

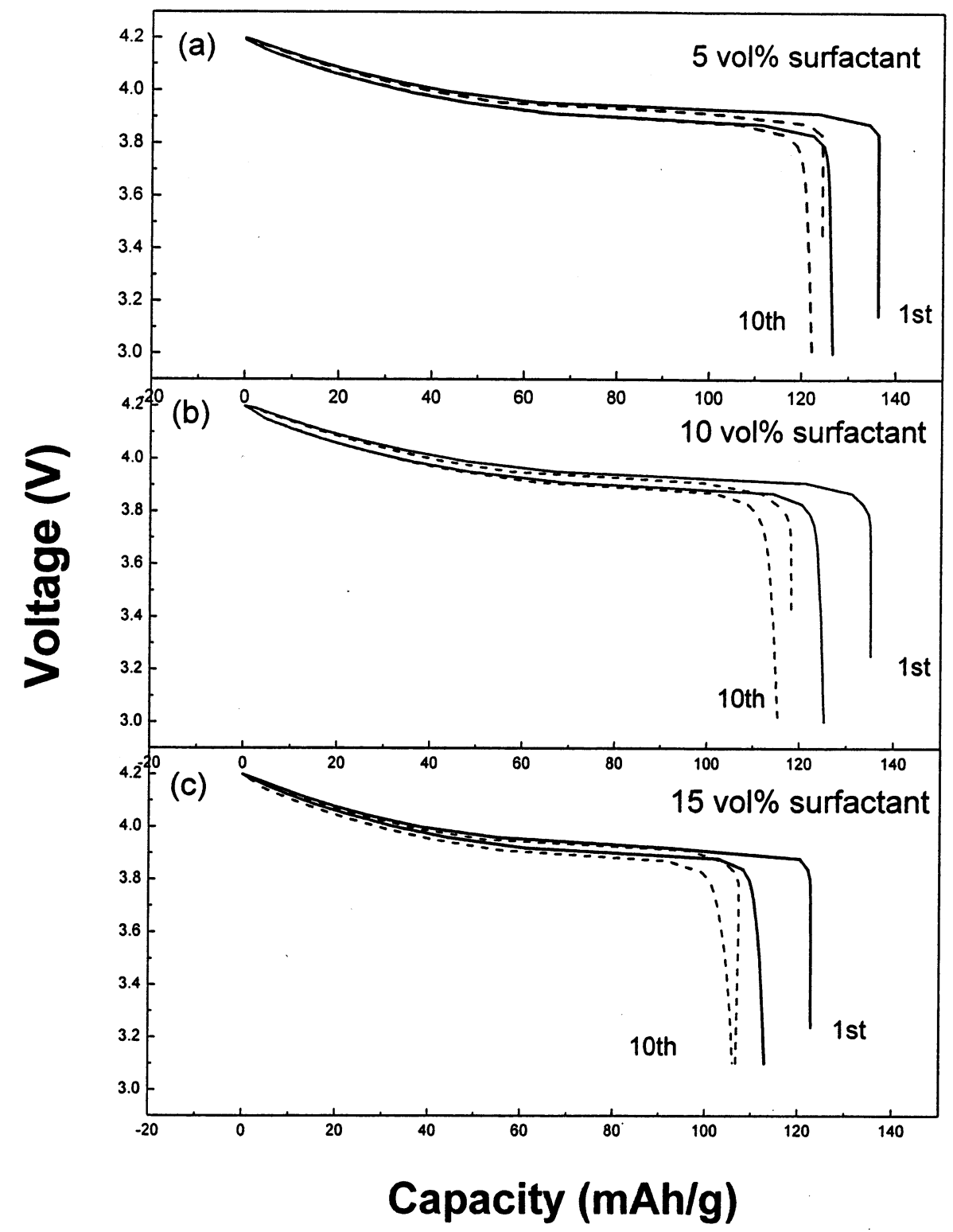

Fig. 3. Charge and discharge curves of $\mathrm{LiCoO}_{2}$ powders derived from the emulsion process using various amounts of the surfactant ((a) 5 vol.\%; (b) 10 vol.\%; and (c) 15 vol. \%). 


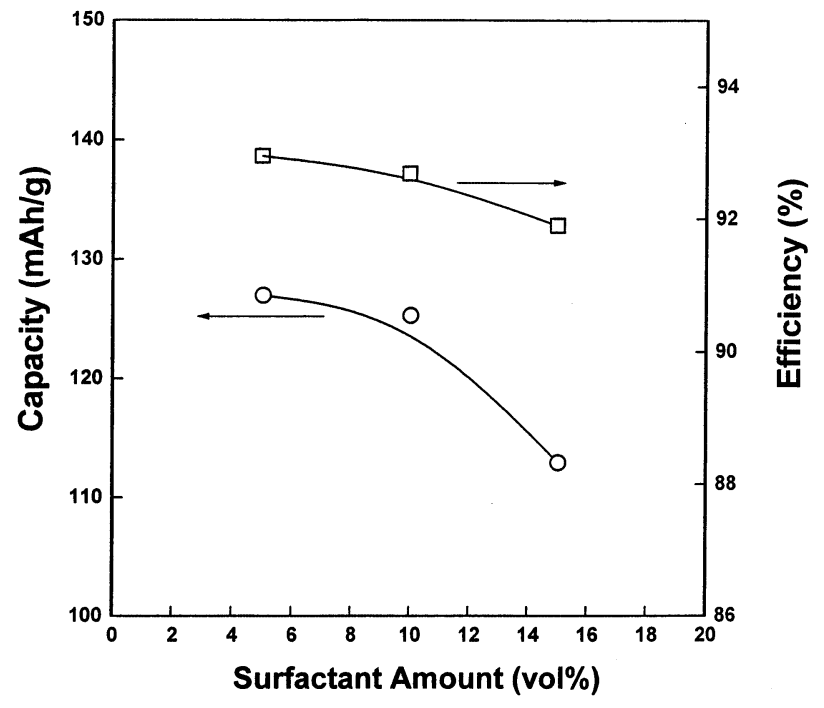

Fig. 4. Coulomb efficiency and discharge capacity of the emulsionderived $\mathrm{LiCoO}_{2}$ as a function of the amount of the surfactant.

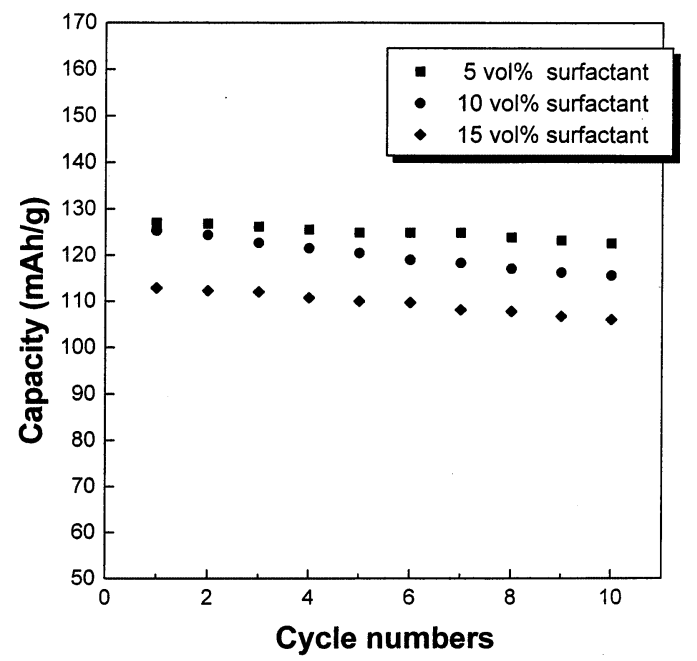

Fig. 5. Discharge capacity versus cycle number for $\mathrm{LiCoO}_{2}$ powders derived from the emulsion process using various amounts of the surfactant.

$P$ is constant. Therefore, when more surfactants are used, the droplet size of emulsion will be decreased, thereby reducing the particle size of the formed $\mathrm{LiCoO}_{2}$ particles. When the amount of the surfactant increases, the interface between the oil and aqueous phases also increases. Since the volume of the aqueous phase used is constant, increasing the interface will result in a decrease in the droplet size in the water-in-oil emulsion. On the other hand, increasing the amount of surfactant was found to reduce the crystallinity of the obtained $\mathrm{LiCoO}_{2}$ powders. According to our earlier study [15], the emulsion-derived precursors consist of $\mathrm{Li}_{2} \mathrm{CO}_{3}$ and $\mathrm{Co}_{3} \mathrm{O}_{4} \cdot \mathrm{Li}_{2} \mathrm{CO}_{3}$ will first liberate carbon dioxide and react with $\mathrm{Co}_{3} \mathrm{O}_{4}$ and $\mathrm{O}_{2}$ to form $\mathrm{LiCoO}_{2}$ at elevated temperatures. During the formation of $\mathrm{LiCoO}_{2}$, the intake of oxygen is required. Increasing the amount of the surfactant is believed to produce a more reducing atmosphere in certain local region due to the thermal decomposition of organic species in surfactants. This reducing atmosphere will hinder the intake of oxygen for the formation of $\mathrm{LiCoO}_{2}$, thereby reducing the crystallinity of the formed particles.

Fig. 3 illustrates the charge-discharge characteristics of $\mathrm{LiCoO}_{2}$ powders obtained by the emulsion process with various amounts of the surfactant added. All obtained powders clearly display a plateau at $3.9 \mathrm{~V}$, which represents the typical electrochemical characteristic of $\mathrm{LiCoO}_{2}$. The coulomb efficiency and discharge capacity of $\mathrm{LiCoO}_{2}$ powders in the first cycle are shown in Fig. 4.

This efficiency is defined as the discharge capacity divided by the charge capacity. It is shown that both the coulomb efficiency and discharge capacity significantly decrease with an increase in the surfactant amount. The first-cycle discharge capacities of $\mathrm{LiCoO}_{2}$ powders prepared with the added amount of the surfactant equal to 5,10 , and $15 \%$ are $126.7,125.3$, and 112.9 $\mathrm{mAh} \mathrm{g}^{-1}$, respectively. The coulomb efficiency also decreases from 93.0 to $91.9 \%$, when the amount of the surfactant increases from 5 to $15 \%$.

It is found that the electrochemical properties of $\mathrm{LiCoO}_{2}$ powders are significantly varied with the amount of the surfactant used. Generally, the crystallinity and particle size of the cathode materials will influence the electrochemical properties. In the present study, the difference in the particle size is not very significant, therefore, the crystallinity of powders seems to play a more important role in affecting the electrochemical properties than the particle size. When the crystallinity of $\mathrm{LiCoO}_{2}$ powders is enhanced with a decrease in the surfactant amount, the disorder of lithium and cobalt cations in the crystal structure will be suppressed, and a more ideal layered structure will be produced. This ideal layered structure facilitates the extraction and intercalation of lithium cations in the host materials, thereby increasing both the discharge capacity and coulomb efficiency. Fig. 5 plots the relation between the discharge capacity and the cycle number. The capacities of all prepared powders only decrease slightly upon cycling. After ten cycles, the discharge capacity still retains about $94-95 \%$ of the value of the first cycle. As a result, $\mathrm{LiCoO}_{2}$ powders with satisfactory cycling characteristics are successfully prepared in this study. However, for obtaining $\mathrm{LiCoO}_{2}$ powders with high specific discharge capacity and coulomb efficiency, the amount of the surfactant has to be well controlled for enhancing the crystallinity and structural order of $\mathrm{LiCoO}_{2}$ powders. 


\section{Conclusions}

Lithium cobalt oxide powders have been prepared by a developed emulsion process. The effects of the surfactant amount used in the emulsion solution on the physical and electrochemical properties of $\mathrm{LiCoO}_{2}$ powders are examined. Well-dispersed monophasic $\mathrm{LiCoO}_{2}$ powders with a layered structure are obtained. Reducing the amount of the surfactant results in improving the crystallinity and slightly decreasing the particle size of $\mathrm{LiCoO}_{2}$ powders. $\mathrm{LiCoO}_{2}$ powders produced from the emulsion solution with a small added amount of the surfactant exhibit high discharge capacity, high coulomb efficiency, and sufficient cyclability.

\section{References}

[1] J.R. Dahn, U. von Sacken, M.W. Juzkown, H. Al-Janaby, J. Electrochem. Soc. 138 (1991) 2207.

[2] T. Ohzuku, A. Ueda, M. Nagayama, J. Electrochem. Soc. 140
(1993) 1862.

[3] W. Ebner, D. Fouchard, L. Xie, Solid State Ionics 69 (1994) 238.

[4] E. Plichta, S. Slane, M. Uchiyama, M. Saloman, D. Chua, W. Ebner, H. Lin, J. Electrochem. Soc. 136 (1989) 1865.

[5] H.F. Gibbard, J. Power Sources 26 (1989) 81.

[6] T. Nagura, K. Tazawa, Prog. Batteries Solar Cells 9 (1990) 20.

[7] R.J. Gummow, M.M. Thackeray, W.I.F. David, S. Hull, Mater. Res. Bull. 27 (1992) 327.

[8] M. Antaya, J.R. Dahn, J.S. Preston, E. Rossen, J.N. Reimers, J. Electrochem. Soc. 140 (1993) 575.

[9] I. Uchida, H. Sato, J. Electrochem. Soc. 142 (1995) L139.

[10] K. Mizushima, P.C. Jones, P.J. Wiseman, J.B. Goodenough, Mater. Res. Bull. 17 (1980) 783.

[11] R.J. Gummow, M.M. Thackeray, W.I.F. David, S. Hull, Mater. Res. Bull. 27 (1992) 327.

[12] Z.S. Peng, C.R. Wan, C.Y. Jiang, J. Power Sources 72 (1998) 215.

[13] E.D. Jeong, M.S. Won, Y.B. Shim, J. Power Sources 70 (1998) 91.

[14] M. Yoshio, H. Tanaka, K. Tominaga, H. Noguchi, J. Power Sources 40 (1992) 347.

[15] C.H. Lu, P.Y. Yeh, J. Mater. Chem. 10 (2000) 599.

[16] Powder Diffraction File, Card No. 44-145. Joint Committee on Powder Diffraction Standards, Swarthmore, PA.

[17] C.H. Lu, C.H. Yeh, Mater. Lett. 33 (1997) 129. 